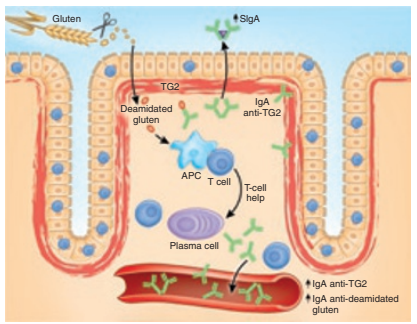

p 3

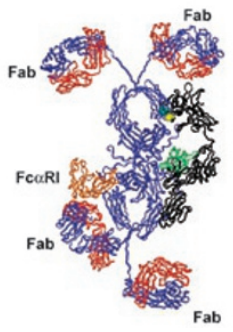

p 74

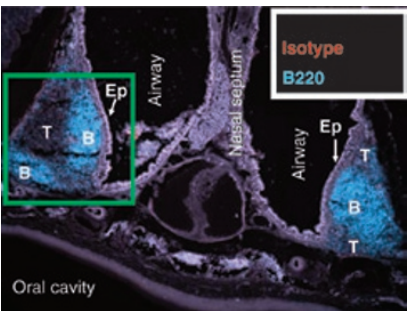

p 85

\section{More than a gluten-free diet?}

In their state-of-the-art Commentary, Sollid and Lundin describe the latest approaches for diagnosing celiac disease and how a greater understanding of disease pathogenesis is helping to advance novel therapeutic strategies for treating patients suffering from this common disorder. See page 3

\section{Pathogenesis of celiac disease}

Meresse and colleagues provide a comprehensive review of celiac disease. They examine the genetic linkage and environmental factors that appear to predispose to disease. Furthermore, they also discuss how loss of tolerance or overreactivity to gluten antigenic epitopes can manifest similarly as food allergy. In addition, the authors report on immune dysregulation seen in celiac patients and discuss whether the alterations in immunoregulation are a cause or an effect of disease. See page 8

\section{Modeling food allergy}

Animal models have been useful for identifying factors that contribute to food allergy. In this review, Berin and Mayer describe the models that have been most useful for understanding the inductive vs. effector phases of sensitivity to food allergens. See page 24

\section{Protecting the barrier}

Schnoor and colleagues identify the GTPase guanylate binding protein-1 as a novel factor that is highly expressed in intestinal epithelial cells from patients with inflammatory bowel disease and that likely helps to preserve intestinal barrier function by blocking interferon- $\gamma$-induced epithelial cell apoptosis. See page 33

\section{Calprotectin protection}

Antimicrobial activity in epithelial cells is mediated in part by calprotectin. Zaia and colleagues explain how Listeria monocytogenes infection of gut epithelial cells can overcome the protection usually afforded by calprotectin. See page 43

\section{Novel peptide vaccines for allergy}

Wells and colleagues describe a novel approach to the suppression of allergic airway inflammation that relies on the use of allergen-specific class I peptide vaccination to drive the induction of counterregulatory CD8-cell responses. The novel vaccine approach appears to elicit a long-lived effect and has demonstrated utility in treating allergic disorders modeled in mice. See page 54

\section{Mucosal vaccination for heterologous nontypeable H. influenzae}

Ostberg and colleagues evaluated immunization strategies for protecting against nontypeable Haemophilus influenzae, an important cause of otitis media and exacerbations of chronic obstructive pulmonary disease in humans. Mucosal, but not systemic, immunization with recombinant outer membrane protein 2 elicited antibodies with reactivity to diverse epitopes on the bacterial surface of multiple bacterial strains. See page 63

\section{Structural insights into the function of SIgA}

Bonner and colleagues provide structural data for human secretory $\lg \mathrm{A} 1$ indicating that this secretory component associates with dimeric $\lg \mathrm{A} 1$ on the $\mathrm{Fc}$ edge. This association preserves the capacity of $\lg A 1$ to bind to FcaR1 receptors, while at the same time masking susceptible sites from bacterial proteases. See page 74

\section{Th1 responses to nasal commensal bacteria}

Costalonga and colleagues describe what happens when commensal or pathogenic bacteria cross the nasal barrier. Although both types of microbes induce surprisingly similar proinflammatory Thelper 1 responses, the response to commensal bacteria is much less efficient and does not result in inflammation. See page 85 